Journal of

\section{Synchrotron}

Radiation

ISSN 0909-0495

Received 24 December 2008

Accepted 24 February 2009

\title{
X-ray diffractometry for the structure determination of a submicrometre single powder grain
}

\author{
Nobuhiro Yasuda, ${ }^{\mathrm{a}, \mathrm{b}}$ Haruno Murayama, ${ }^{\mathrm{c}}$ Yoshimitsu Fukuyama, ${ }^{\mathrm{a}, \mathrm{b}}$ Jungeun Kim, ${ }^{\mathrm{a}, \mathrm{b}}$ \\ Shigeru Kimura, ${ }^{\mathrm{a}, \mathrm{b} *}$ Koshiro Toriumi, ${ }^{\mathrm{b}, \mathrm{d}}$ Yoshihito Tanaka, ${ }^{\mathrm{b}, \mathrm{e}}$ Yutaka Moritomo, \\ Yoshihiro Kuroiwa, ${ }^{b, g}$ Kenichi Kato, ${ }^{b, e}$ Hitoshi Tanaka ${ }^{b, e}$ and Masaki Takata ${ }^{a, b, e, h}$ \\ aJASRI/SPring-8, 1-1-1 Kouto, Sayo-cho, Sayo-gun, Hyogo 679-5198, Japan, bJapan Science and \\ Technology Agency, CREST, 5 Sanbancho, Chiyoda-ku, Tokyo 102-0075, Japan, 'Department of \\ Applied Chemistry, Faculty of Science and Engineering, Chuo University, 1-13-27 Kasuga, Bunkyo- \\ ku, Tokyo 112-8551, Japan, ${ }^{\mathbf{d}}$ Graduate School of Material Science, University of Hyogo, 3-2-1 \\ Kouto, Kamigori-cho, Ako-gun, Hyogo 678-1297, Japan, ${ }^{\mathbf{e}}$ RIKEN SPring-8 Center, Harima Institute, \\ 1-1-1 Kouto, Sayo-cho, Sayo-gun, Hyogo 679-5148, Japan, ${ }^{\mathbf{f}}$ The Graduate School of Pure and \\ Applied Sciences, University of Tsukuba, Tenodai, Tsukuba, Ibaraki 305-8571, Japan, ${ }^{\mathbf{g}}$ Department \\ of Physical Science, Graduate School of Science, Hiroshima University, 1-3-1 Kagamiyama, \\ Higashi-Hiroshima, Hiroshima 739-8526, Japan, and ${ }^{\mathbf{h}}$ Department of Advanced Materials Science, \\ School of Frontier Sciences, The University of Tokyo, 5-1-5 Kashiwanoha, Kashiwa-shi, Chiba \\ 277-8561, Japan. E-mail: kimuras@spring8.or.jp
}

\begin{abstract}
A high-precision diffractometer has been developed for the structure analysis of a submicrometre-scale single grain of a powder sample at the SPring-8 BL40XU undulator beamline. The key design concept is the combination of a stable focused synchrotron radiation beam and the precise axis control of the diffractometer, which allows accurate diffraction intensity data of a submicrometre-scale single powder grain to be measured. The phase zone plate was designed to create a high-flux focused synchrotron radiation beam. A loweccentric goniometer and high-precision sample positioning stages were adopted to ensure the alignment of a micrometre-scale focused synchrotron radiation beam onto the submicrometre-scale single powder grain. In order to verify the diffractometer performance, the diffraction pattern data of several powder grains of $\mathrm{BaTiO}_{3}$, of dimensions $\sim 600 \times 600 \times 300 \mathrm{~nm}$, were measured. By identifying the diffraction data set of one single powder grain, the crystal structure was successfully determined with a reliable factor of $5.24 \%$.
\end{abstract}

Keywords: submicrometre X-ray beam; phase zone plate; X-ray diffraction; single-crystal structure analysis; powder diffraction.

\section{Introduction}

Up to now, the structure identification and characterization of various new materials have been carried out by powder diffraction experiments as newly synthesized materials are, in most cases, obtained in powder and/or in polycrystalline forms. This research technical trend has been accelerated by the highphoton-flux monochromatic X-rays of synchrotron radiation in addition to the progress of computers and software for structure determination. Consequently, the development of an $a b$ initio structure analysis technique, which denotes unknown structure determination, is now one of the most topical research subjects in powder crystallography (David et al., 2002; David \& Shankland, 2008). On the other hand, an X-ray synchrotron radiation beam with high brilliance and small divergence allows an alternative method to be suggested, which can determine the crystal structure of a micrometre- or submicrometre-scale single crystal corresponding to a single grain of a powder sample. In fact, some structure determinations of a few micrometre-sized single crystals were recently reported using a synchrotron radiation focusing technique (Riekel et al., 2005; Volkringer et al., 2007). Before those works, the Laue method, which uses an intense white synchrotron radiation beam instead of a focused monochromatic beam, was also performed on a submicrometre crystal to measure the intensity data efficiently (Ohsumi et al., 1991). However, the conventional structure determination of a submicrometre-scale single crystal has not yet been reported. This is mainly due to three technical difficulties which must be overcome: the accurate intensity data collection of very weak diffraction spots, the precise centring of the invisible sample, and the infallible manipulation of a submicrometre-scale 
single crystal. The combination of the synchrotron radiation focusing technique and the precise axis control of the diffractometer is a solution for the first and second difficulties. The latest focusing technique for a synchrotron radiation diffraction experiment can firmly produce a micrometre-scale high-flux synchrotron radiation beam. The precise axis control enables the submicrometre-scale sample to be moved and kept within the micrometre-scale focused synchrotron radiation beam. We have therefore developed and optimized a highprecision diffractometer which combines a synchrotron radiation focusing technique and low eccentric sample rotation at the SPring-8 BL40XU undulator beamline, to enable the structure analysis of a submicrometre-scale single powder grain. As for the third difficulty, since the development of the systematic manipulation techniques to select and capture one single grain of powder sample is still in progress, several submicrometre-scale single powder grains have been attached to a fine glass fibre by means of the conventional sample manipulation technique.

From a technical viewpoint, the structure analysis of a submicrometre-scale single powder grain has great advantages for applications to structural materials science research. Firstly, we can remove the intrinsic problem in the powder diffraction method, peak overlapping, which causes ambiguities in the determined structure in most cases, since a single powder grain can be mostly treated as a single crystal. Secondly, submicrometre-scale samples enable us to measure data free from extinction and/or absorption correction.

Another advantage arises in the case of observations of photo-induced phase transition (PIPT). For laser-pump and synchrotron-radiation-probe experiments, we can avoid the mismatch between the penetration depths of laser and $\mathrm{X}$-rays since a typical penetration depth of laser light is of the order of $1 \mu \mathrm{m}$. This indicates near $100 \%$ yield of PIPT, which greatly improves the accuracy of the structure analysis of PIPT, is achievable with a submicrometre-scale single powder grain.

Practically, the structure analysis of a submicrometre-scale single powder grain can be directly applied to the research of the grain-size-dependent structure-property relationship. One of the most attractive examples is a barium titanate $\left(\mathrm{BaTiO}_{3}\right)$ fine particle, which shows a phase transition from tetragonal to cubic as the particle size decreases. Some diffraction studies of the size effect of $\mathrm{BaTiO}_{3}$ were carried out using the powder diffraction method (Aoyagi et al., 2002). However, the powder diffraction method had the following disadvantages: the obtained structural parameters are the averaged information from various size particles in the powder sample; in addition, the peak overlapping becomes more serious owing to the peak profile broadening caused by the small particle size. The structure analysis of a single powder grain could overcome these difficulties because the diffraction peaks are isolated geometrically.

In this article we illustrate the concepts and features of the high-precision diffractometer for the structure analysis of a single powder grain, with an example of the structure analysis of a $\mathrm{BaTiO}_{3}$ fine particle of dimensions $\sim 600 \times 600 \times 300 \mathrm{~nm}$.

\section{Fundamental design of the high-precision diffractometer}

The following qualifications are essential to complete the highprecision diffractometer: (i) high photon flux density of incident synchrotron radiation beam; (ii) low background intensity; (iii) easy alignment and high-performance focusing system; (iv) stable and precise axis rotation of the diffractometer.

Regarding qualifications (i) and (ii), we installed the diffractometer with the synchrotron radiation focusing system at the SPring-8 BL40XU helical undulator beamline (Inoue $e t$ al., 2001; Kimura et al., 2007). Fig. 1(a) shows the experimental set-up of the high-precision diffractometer system, and Fig. 1(b) is a photograph of the high-precision diffractometer. Fine focusing of the synchrotron radiation beam enables us to produce the incident synchrotron radiation beam with high photon flux density and small beam size, which improves the signal-to-background ratio because most of the focused synchrotron radiation beam can be incident on the sample.

For qualification (iii), we adopted a phase zone plate to focus the synchrotron radiation beam as on-axis focusing is convenient for diffraction measurements and tuning the focused beam size is easy by adjusting the longitudinal position of the phase zone plate.

Qualification (iv) is key for the structure analysis of a submicrometre-scale single powder grain. The diffraction data are measured in the oscillation mode, which is the same as in single-crystal structure analysis. In order to collect sufficiently
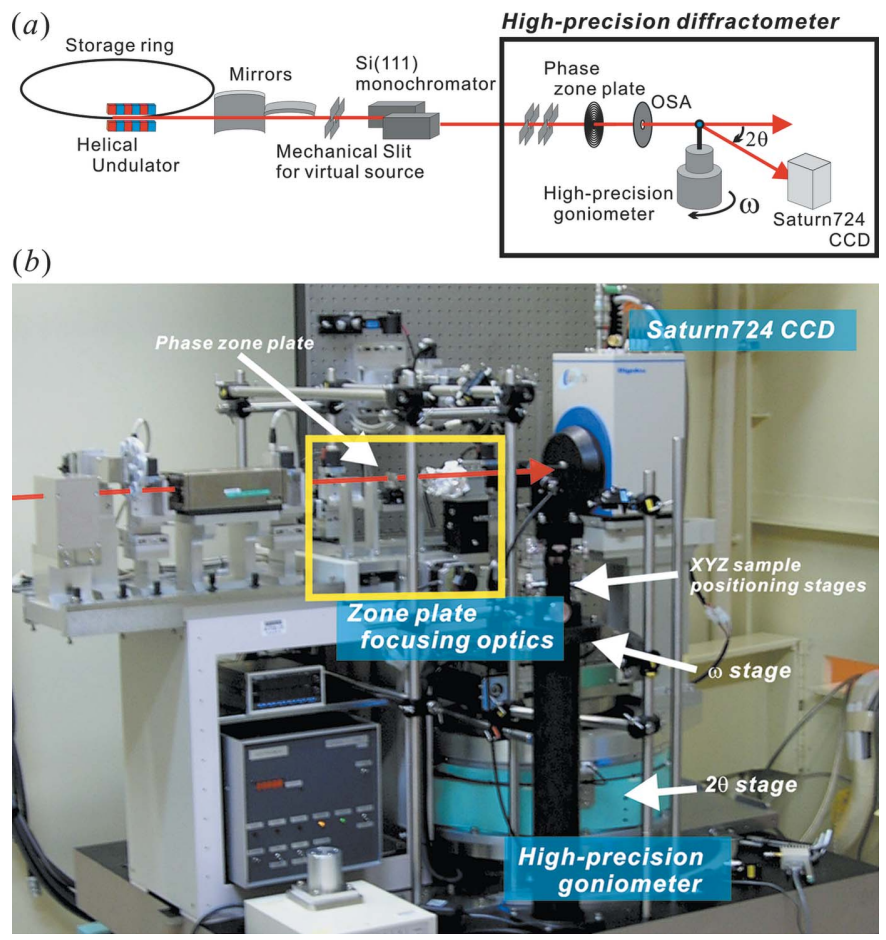

Figure 1

(a) Schematic diagram of the experimental set-up of the high-precision diffractometer system. (b) Photograph of the high-precision diffractometer. The red arrow and yellow box indicate the synchrotron radiation beam and the zone plate focusing optics, respectively. 
accurate diffraction data, the submicrometre-scale single powder grain must be kept within the focused X-ray beam during the measurement. To this end, high-precision $\omega$ and $2 \theta$ rotation stages were installed in the diffractometer. To avoid eccentric rotation owing to gravitational influences on the rotation axis, vertical $\omega$ and $2 \theta$ rotation axes were adopted. The vertical rotation axis is not usually used for synchrotron radiation diffraction experiments because of horizontal polarization of the synchrotron radiation beam. However, a helical undulator, which radiates the circularly polarized synchrotron radiation beam, enabled us to use the vertical axis rotation stages.

Another advantage of the helical undulator is that the higher harmonics are emitted towards an off-axial beam direction. By extracting the central part of the radiation, therefore, the high-photon-flux beam $\left(\sim 10^{15}\right.$ photons s $\left.^{-1}\right)$ with narrow peak-energy width $(\Delta E / E \simeq 2 \%)$ can be used for quasi-monochromatic synchrotron radiation $\mathrm{X}$-rays. The fundamental radiation can be selected from 8 to $17 \mathrm{keV}$ by changing the undulator gap. A further monochromated synchrotron radiation beam $(\Delta E / E \simeq 0.02 \%)$ is made using a $\mathrm{Si}(111)$ channel-cut monochromator.

\subsection{Zone plate focusing optics}

We designed two different phase zone plates (ZP1 and ZP2), fabricated by the NTT-AT Nanofabrication Corporation (Ozawa et al., 1997). ZP1 is designed for experiments preferring higher photon energy and high photon flux density such as the structure analysis of a submicrometre-scale single powder grain. On the contrary, ZP2 is designed for experiments such as scanning $\mathrm{X}$-ray microscopy requiring high spatial resolution.

$\mathrm{ZP} 1$ has a diameter of $100 \mu \mathrm{m}$, an innermost zone radius of $5.0 \mu \mathrm{m}$, an outermost zone width of $250 \mathrm{~nm}$ and a tantalum thickness of $2.5 \mu \mathrm{m}$. The thickness was designed to have maximum diffraction efficiency at $15 \mathrm{keV}(\sim 26 \%)$. On the other hand, ZP2 has a diameter of $120 \mu \mathrm{m}$, an innermost zone radius of $3.0 \mu \mathrm{m}$, an outermost zone width of $75 \mathrm{~nm}$ and a tantalum thickness of $750 \mathrm{~nm}$. The ideal diffraction efficiency is $12 \%$ at $8 \mathrm{keV}$. The focal lengths of $\mathrm{ZP} 1$ and $\mathrm{ZP} 2$ are 300 and $50 \mathrm{~mm}$ for 15 and $8 \mathrm{keV}$, respectively.

Fig. 2 shows details of the zone plate focusing optics, which has two independent $X Y Z$ positioning stages that align the positions of the phase zone plate and the order-sorting aperture (OSA). To align the sample onto the focused synchrotron radiation beam, an optical microscope (KEYENCE VHZ100) with a CCD video camera can be inserted on the beam axis with a translation stage (stage $x$ ) on which the zone plate optics and the optical microscope are mounted in parallel. The optical microscope has a working distance of $25 \mathrm{~mm}$ and a magnification range from 100 to 1000 , and a few micrometresized samples can be observed on a TV monitor. The position stability and reproducibility of the zone plate optics and the optical microscope are monitored by the linear gauge with $100 \mathrm{~nm}$ resolution.

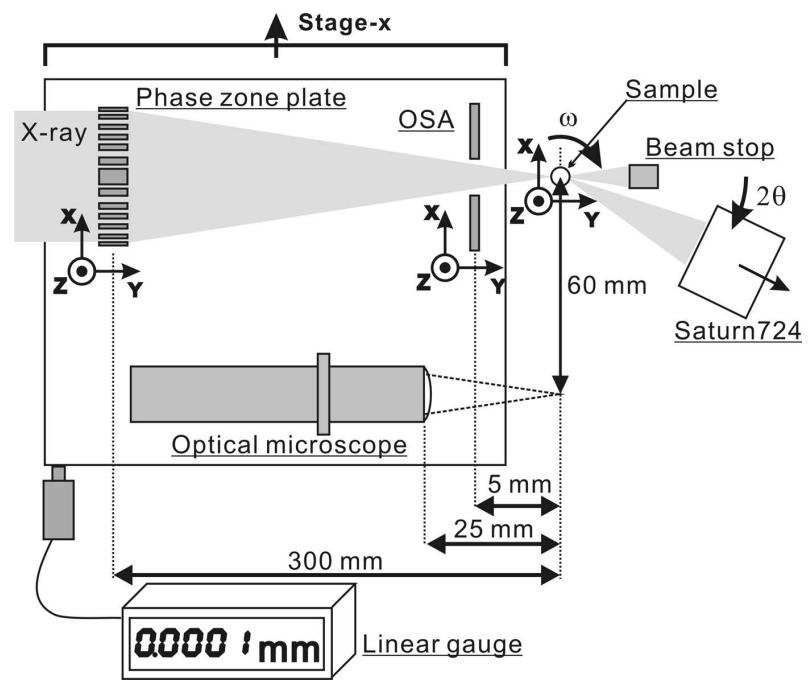

Figure 2

Schematic diagram of the zone plate focusing optics. The phase zone plate and OSA are aligned by the $X Y Z$ positioning stages. These components are mounted on the same translation stage (stage $x$ ), which can be moved by $60 \mathrm{~mm}$ on the aligned light axis to enable sample centring using the optical microscope. The position repeatability of stage $x$ is monitored by the linear gauge with $100 \mathrm{~nm}$ resolution.

\subsection{High-precision goniometer}

The high-precision goniometer has $\omega-2 \theta$ rotation and $X Y Z$ sample positioning stages (Fig. 2). Highly accurate reproducibility of the rotation centre is required for these stages to keep the micrometre-scale focused X-ray beam on the submicrometre-scale single powder grain during a measurement. We therefore adopted a high-precision air-bearing stage (Canon AB-100R) to achieve low eccentric $\omega$-rotation within $\pm 100 \mathrm{~nm}$ per $360^{\circ}$. The $X Y Z$ sample positioning stages also have $5 \mathrm{~nm}$ resolution.

Since the development of the sample manipulation technique of invisible submicrometre-scale powder grains has not yet been completed, several grains are presently attached to the tip of a glass fibre with a diameter of a few micrometres. The centring of the sample can be carried out systematically by using the tip of a glass fibre, not the grains, as a target. Because the diameter of the glass fibre is a few micrometres, the sample alignment can be controlled to less than $1 \mu \mathrm{m}$ using the large magnification optical microscope inserted on the beam axis.

\subsection{X-ray CCD detector}

A high-sensitive and low-noise X-ray CCD detector (Rigaku Saturn724), having a $72 \times 72 \mathrm{~mm}$ image area, and outputting 16-bit $2080 \times 2080$ pixel two-dimensional data, was used to detect weak diffraction spots of the submicrometrescale single powder grains. The X-ray CCD is mounted on the arm of the $2 \theta$ rotation stage with a translation stage to change the camera distance from 30 to $135 \mathrm{~mm}$. The $2 \theta$ resolution can be selected from $0.065^{\circ}$ to $0.015^{\circ}$ per pixel. The interval of the measurement owing to the dead time of the CCD camera and $\omega$-axis positioning was about $15 \mathrm{~s}$. As a result, the measure- 
Table 1

Achieved performances of phase zone plates ZP1 and ZP2.

\begin{tabular}{|c|c|c|}
\hline & ZP1 & $\mathrm{ZP2}$ \\
\hline Photon energy (keV) & 15 & 8 \\
\hline Beam size, vertical $\times$ horizontal $(\mu \mathrm{m})$ & $1.4 \times 2.9$ & $0.33 \times 0.47$ \\
\hline Photon flux (photons s ${ }^{-1}$ ) & $1.25 \times 10^{10}$ & $2.92 \times 10^{8}$ \\
\hline Photon flux density (photons $\mathrm{s}^{-1} \mu \mathrm{m}^{-2}$ ) & $3.1 \times 10^{9}$ & $1.9 \times 10^{9}$ \\
\hline Gain factor & 400 & 4400 \\
\hline
\end{tabular}

ment for a data set takes less than $1 \mathrm{~h}$ for a micrometre-scale crystal and a few hours for a submicrometre-scale crystal. Rigaku's Rapid Auto System (Rigaku, 1998) is available for processing the measured diffraction data.

\section{Beam size and photon flux on the sample position}

The synchrotron radiation beam is pre-focused by two mirrors that are installed in the optics hutch. The focal point of the mirrors is located as the virtual source at $8900 \mathrm{~mm}$ upstream of the phase zone plate (Fig. 1a). The size of the virtual source can be tuned to $5 \times 5 \mu \mathrm{m}$ by the mechanical slit.

Without the mechanical slit shaping of the virtual source, the photon flux densities of the $15 \mathrm{keV}$ and $8 \mathrm{keV}$ synchrotron radiation beams in front of the phase zone plate position were $7.9 \times 10^{6}$ and $4.3 \times 10^{5}$ photons $\mathrm{s}^{-1} \mu \mathrm{m}^{-2}$, respectively. The synchrotron radiation beam is focused by the phase zone plate, and the first-order focused synchrotron radiation beam is only selected with the OSA placed before the sample position. The horizontal and vertical beam sizes at the focal position (i.e. the sample position) were evaluated by a knifeedge scan method using Au meshes. The beam profiles in the vertical direction at the focal position are shown in Fig. 3. Achieved performances of ZP1 and ZP2 are listed in Table 1. In the present study we achieved beam sizes of 1.4 (vertical) $\times$ 2.9 (horizontal) $\mu \mathrm{m}$ for $15 \mathrm{keV}$ with $\mathrm{ZP} 1$ and $330 \times 470 \mathrm{~nm}$ for $8 \mathrm{keV}$ with ZP2. The photon flux densities increased to $3.1 \times$ $10^{9}$ and $1.9 \times 10^{9}$ photons $\mathrm{s}^{-1} \mu \mathrm{m}^{-2}$. The corresponding gain factors are almost 400 and 4400, respectively. The angular divergence of the focused beam was increased to $0.019^{\circ}$ for $15 \mathrm{keV}$ with $\mathrm{ZP} 1$ and $0.13^{\circ}$ for $8 \mathrm{keV}$ with $\mathrm{ZP} 2$. Thus, in the

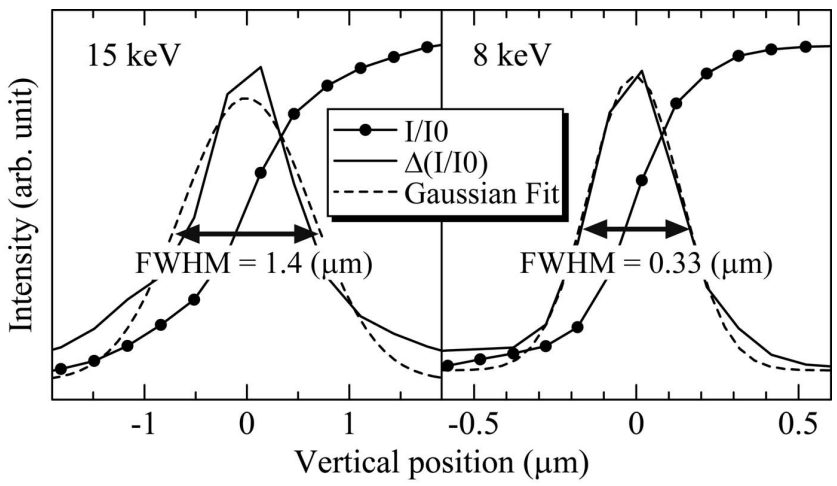

Figure 3

Knife-edge scan intensity data of the 15 and $8 \mathrm{keV}$ microfocusing synchrotron radiation beams in the vertical direction at the focal position (solid circles). Solid and broken lines are derivatives of the measured intensity and the Gaussian fitting profiles, respectively. case of $\mathrm{ZP} 2$, the effect of the data resolution on structure analysis should be taken into account. It should be noted that further focused beam was available by reducing the virtual source size, even though the flux becomes extremely low. The ultimate beam size of the present system was $79 \times 87 \mathrm{~nm}$ at $8 \mathrm{keV}$ with a $10 \times 10 \mu \mathrm{m}$ virtual source size. The photon flux decreased to $1.51 \times 10^{7}$ photons $\mathrm{s}^{-1}$, while the photon flux density increased to $2.19 \times 10^{9}$ photons s${ }^{-1} \mu^{-2}$.

\section{Structure analysis of a submicrometre-scale $\mathrm{BaTiO}_{3}$ single powder grain}

Structure analysis was performed for a submicrometre-scale $\mathrm{BaTiO}_{3}$ single powder grain. The grains of $\mathrm{BaTiO}_{3}$ were attached to the tip of a fine glass fibre with epoxy adhesive by means of an optical microscope and a micromanipulator. Figs. 4(a) and 4(b) show the optical microscope and scanning electron microscope (SEM) images of the tip of a fine glass fibre, respectively. Several $\mathrm{BaTiO}_{3}$ grains were attached to the glass fibre. The maximum grain size was about $600 \times 600 \times$ $300 \mathrm{~nm}$.

The diffraction data were collected in the $\omega$-oscillation mode at room temperature. The $15 \mathrm{keV}$ focused synchrotron radiation beam was prepared by $\mathrm{ZP} 1$. The calibration of the wavelength was carried out using the powder diffraction pattern of a NIST (National Institute of Standards and Technology) X-ray standard sample, $\mathrm{CeO}_{2}$ (Standard Reference Material 674a). The exposure time and the $\omega$-axis scan step, $\Delta \omega$, were $15 \mathrm{~s}$ and $1^{\circ}$, respectively. With this set-up, the 360 diffraction images were collected in $180 \mathrm{~min}$. The overlapped diffraction pattern of the 360 images is shown in Fig. 4(c). The shape of the diffraction spots does not represent significant deformation caused by the strain of the sample adhered on the glass fibre. The accidentally diffracted spots of the other $\mathrm{BaTiO}_{3}$ grains sometimes interfered in the diffraction pattern during $\omega$-oscillation. These extra spots can be discriminated systematically because the diffraction pattern no longer shows the Debye-Scherrer pattern but the superposition of several single-crystal diffraction patterns. At this moment, however, it is very difficult to directly identify the sample grain corresponding to the diffraction spots among the several sample grains (Fig. $4 b$ ).

The crystal structure was solved by direct methods (SHELXS97) and refined by full-matrix least-squares on $F^{2}$ (SHELXL97) (Sheldrick, 2008). Only the thermal vibration of the $\mathrm{Ba}$ atoms was refined anisotropically. The reliability factor of the refinement finally became $R_{1}=5.24 \%$. The crystal structure and the determined crystallographic parameters are shown in Fig. 4(c) and Table 2, respectively. ${ }^{1}$

The crystal system of a $600 \times 600 \times 300 \mathrm{~nm} \mathrm{BaTiO}{ }_{3}$ single powder grain at room temperature was finally determined to be tetragonal. The values of the cell parameters, $a=$ $3.9905(13) \AA$ and $c=4.0412(14) \AA$, correspond to those

\footnotetext{
${ }^{1}$ Supplementary data for this paper are available from the IUCr electronic archives (Reference: IA5038). Services for accessing these data are described at the back of the journal.
} 

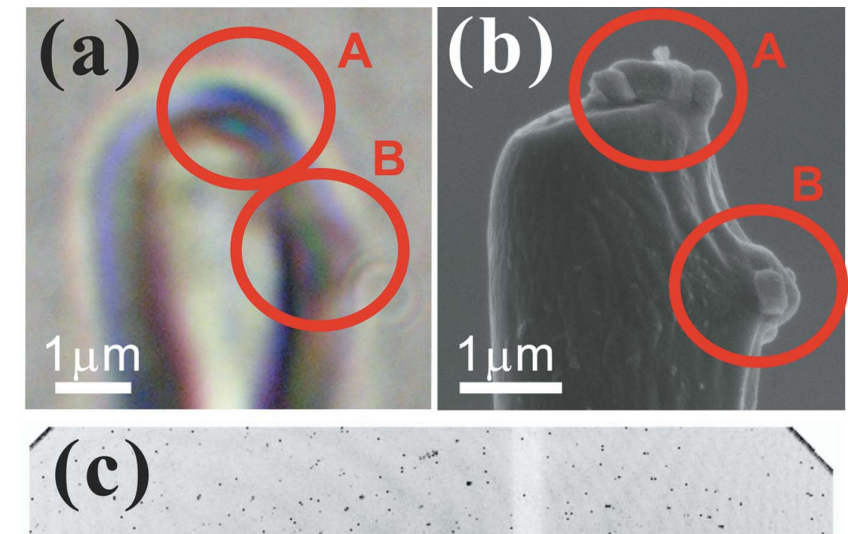

a

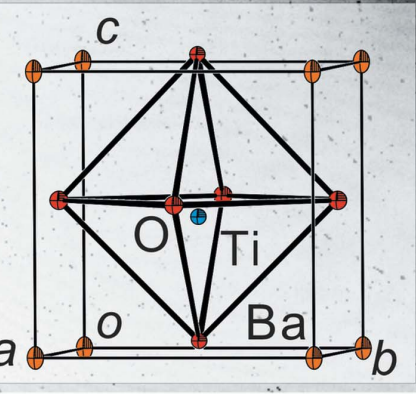

Figure 4

Photographs of $\mathrm{BaTiO}_{3}$ powder grains attached to the tip of the fine glass fibre: (a) optical-microscope image. (b) SEM image of the same sample. The size of the large grain is about $600 \times 600 \times 300 \mathrm{~nm}$. A and B in each figure show the positions of existing $\mathrm{BaTiO}_{3}$ grains. (c) Measured diffraction image of $\mathrm{BaTiO}_{3}$ grains. All measured images are overlapped on one image to see Bragg diffraction spots clearly. The vertical and horizontal $2 \theta$ range of the image are $\pm 45^{\circ}$ and $-30-60^{\circ}$, respectively. The inset is a $50 \%$ probability level ORTEP image of the refined $\mathrm{BaTiO}_{3}$ crystal structure.

derived from the powder diffraction studies of $a=$ 3.9950 (1) $\AA$ and $c=4.0340$ (1) $\AA$ for $400 \mathrm{~nm}$-sized particles (Aoyagi et al., 2002) and 3.99900 (8) $\AA$ and 4.03265 (9) $\AA$ for $430 \mathrm{~nm}$-sized particles (Yashima et al., 2005), respectively. This correspondence of the present result with the powder diffraction results proves that the developed diffractometry has great potential for revealing the one-on-one relationship between the crystal size and the crystal structure, if we realise the structure analysis of a single powder grain smaller than $100 \mathrm{~nm}$.

\section{Conclusion}

The crystal structure of a submicrometre-scale $\mathrm{BaTiO}_{3}$ single powder grain was successfully determined for several single powder grains. This success proves that the diffraction measurement of a single powder grain has a sufficient advantage in the determination of unknown crystal structure
Table 2

Crystal data and experimental details for $\mathrm{BaTiO}_{3}$.

\begin{tabular}{ll}
\hline Formula & $\mathrm{BaTiO}_{3}$ \\
Formula weight & 233.24 \\
Temperature $(\mathrm{K})$ & $300(2)$ \\
Wavelength $(\AA)$ & 0.83351 \\
Crystal system & Tetragonal \\
Space group & $P 4 m m$ \\
$a(\AA)$ & $3.9905(13)$ \\
$b(\AA)$ & $3.9905(13)$ \\
$c(\AA)$ & $4.0412(14)$ \\
Volume $\left(\AA^{3}\right)$ & $64.35(4)$ \\
$Z$ & 1 \\
$D_{\text {calc }}\left(\mathrm{Mg} \mathrm{m}^{-3}\right)$ & 6.018 \\
Absorption coefficient $\left(\mathrm{mm}^{-1}\right)$ & 18.037 \\
Crystal size $(\mathrm{nm})$ & $600 \times 600 \times 300$ \\
$\theta$ range $\left({ }^{\circ}\right)$ & $5.92-29.26$ \\
Resolution $(\AA)$ & $4.04-0.85$ \\
Reflections collected/unique & $468 / 88$ \\
$R_{\text {int }}$ & 0.0940 \\
Completeness to $\theta_{\text {max }}$ & 1.00 \\
Data/restraints $/$ parameters & $88 / 0 / 9$ \\
$R_{1}[I>2 \sigma(I)]$ & 0.0524 \\
$R_{w}[I>2 \sigma(I)]$ & 0.0994 \\
Goodness of fit & 1.168 \\
Number of $I>2 \sigma(I)$ & 85 \\
Largest diffraction peak $\left(\mathrm{e} \AA^{-3}\right)$ & 2.079 \\
Largest diffraction hole $\left(\mathrm{e} \AA^{-3}\right)$ & -1.980 \\
\hline
\end{tabular}

for any powder-formed crystalline samples. Thus, the structure analysis of a single powder grain shall become an alternative method of powder diffraction structure analysis.

Note that the present result was obtained from one of the several grains attached on a glass fibre. Thus the manipulation technique to select only one powder grain is the remaining difficulty to be solved.

We have also developed a laser-pump and synchrotronradiation-probe diffraction technique using the same diffractometer system (Fukuyama et al., 2008a,b). Now we are ready to apply the structure analysis of a single powder grain for the research of the photo-induced phase transition combined with the pump and probe technique.

We thank all the members of the X-ray pinpoint structure measurement group for their helpful discussions. We also thank Professor Satoshi Wada for the $\mathrm{BaTiO}_{3}$ sample preparation, and Professor Shinji Matsui and Mr Makoto Okada for the SEM measurement. Part of this research was supported by a Grant-in-Aid for Young Scientists (B) (No. 18750023) from the Japanese Ministry of Education, Culture, Sports, Science and Technology. This work was conducted at the BL40XU of SPring-8 with the approval of the Japan Synchrotron Radiation Research Institute (Proposal Nos. 2005A0885-S3-np, 2005B0930, 2006A1746, 2006B1726, 2007A1864 and 2007B1876).

\section{References}

Aoyagi, S., Kuroiwa, Y., Sawada, A., Yamashita, I. \& Atake, T. (2002). J. Phys. Soc. Jpn, 71, 1218-1221.

David, W. I. F. \& Shankland, K. (2008). Acta Cryst. A64, 52-64.

David, W. I. F., Shankland, K., McCusker, L. B. \& Baerlocher, Ch. (2002). Editors. Structure Determination from Powder Diffraction Data, pp. 1-11. Oxford University Press. 
Fukuyama, Y., Yasuda, N., Kim, J. E., Murayama, H., Ohshima, T., Tanaka, Y., Kimura, S., Kamioka, H., Moritomo, Y., Toriumi, K, Tanaka, H., Kato, K., Ishikawa, T. \& Takata, M. (2008a). Rev. Sci. Instrum. 79, 045107.

Fukuyama, Y., Yasuda, N., Kim, J. E., Murayama, H., Tanaka, Y., Kimura, S., Kato, K., Kohara, S., Moritomo, Y., Matsunaga, T., Kojima, R., Yamada, N., Tanaka, H., Ohshima, T. \& Takata, M. (2008b). Appl. Phys. Express, 1, 045001.

Inoue, K., Oka, T., Suzuki, T., Yagi, N., Takeshita, K., Goto, S. \& Ishikawa, T. (2001). Nucl. Instrum. Methods Phys. Res. A, 467-468, 674-677.

Kimura, S., Moritomo, Y., Tanaka, Y., Tanaka, H., Toriumi, K., Kato, K., Yasuda, N., Fukuyama, Y., Kim, J. E., Murayama, H. \& Takata, M. (2007). AIP Conf. Proc. 879, 1238-1241.
Ohsumi, K., Hagiya, K. \& Ohmasa, M. (1991). J. Appl. Cryst. 24, 340348.

Ozawa, A., Tamamura, T., Ishii, T., Yoshihara, H. \& Kagoshima, Y. (1997). Microelectron. Eng. 35, 525-529.

Riekel, C., Burghammer, M. \& Schertler, G. (2005). Curr. Opin. Struct. Biol. 15, 556-562.

Rigaku Corporation (1998). RAPID-AUTO. Rigaku Corporation, Tokyo, Japan.

Sheldrick, G. M. (2008). Acta Cryst. A64, 112-122.

Volkringer, C., Popov, D., Loiseau, T., Guillou, N., Ferey, G., Haouas, M., Taulelle, F., Mellot-Draznieks, C., Burghammer, M. \& Riekel, C. (2007). Nat. Mater. 6, 760-764.

Yashima, M., Hoshina, T., Ishimura, D., Kobayashi, S., Nakamura, W., Tsurumi, T. \& Wada, S. (2005). J. Appl. Phys. 98, 014313. 